Bull. Austral. Math. Soc.

46в $20,46 \mathrm{~B} 08$

VoL. 67 (2003) [225-240]

\title{
GENERALISED JORDAN-VON NEUMANN CONSTANTS AND UNIFORM NORMAL STRUCTURE
}

\author{
S. Dhompongsa, P. Piraisangunn and S. Saejung
}

We introduce a new geometric coefficient related to the Jordan-von Neumann constant. This leads to improved versions of known results and yields new ones on super-normal structure for Banach spaces.

\section{INTRODUCTION}

The notions of normal structure and uniform normal structure play an important role in metric fixed point theory (see Goebel and Kirk [10]). A number of Banach space properties have been shown to imply uniform normal structure. Some sufficient properties for a Banach space $\mathrm{X}$ to have uniform normal structure are:

(i) $J(X)<3 / 2$ (see Gao and Lau [6]),

(ii) $R(X)>0$ (see Gao [5]),

(iii) $C_{\mathrm{NJ}}(X)<5 / 4$ (see Kato, Maligranda and Takahashi [13]), and

(iv) $X$ is a u-space, a class of spaces that includes uniformly convex spaces and uniformly smooth spaces (see Gao and Lau [6]).

Recently, Kirk and Sims [17] introduced a new variant, $\phi$-uniform normal structure, which lies strictly between normal structure and uniform normal structure.

In this paper we introduce a parameterised coefficient $C_{\mathrm{NJ}}(\cdot, X)$ generalising the Jordan-von Neumann constant $C_{\mathrm{NJ}}(X)$. Utilising ultraproduct techniques, the coefficient $C_{\mathrm{NJ}}(\cdot, X)$ enables us to establish new sufficient conditions for a Banach space to have uniform normal structure. To achieve this, we first show that the coefficients $C_{\mathrm{NJ}}(\cdot, X)$ of the space $\mathrm{X}$ and $C_{\mathrm{NJ}}(\cdot, \tilde{X})$ of its ultrapower $\widetilde{X}$ coincide. From this and some other new results, which also improve the number appearing in property (iii) from $5 / 4$ to $(3+\sqrt{5}) / 4$, we can apply the powerful ultraproduct technique to show that $\mathrm{X}$ has uniform normal structure whenever $C_{\mathrm{NJ}}(1, X)<2$. An example of a Banach space $X$ is given which has $C_{\mathrm{NJ}}(1, X)<2$ and hence uniform normal structure, but for which neither (i) or (iii) apply. An exact determination of the coefficient $C_{\mathrm{NJ}}(\cdot, X)$ is obtained when $\mathrm{X}$ is a Hilbert space. More generally, a connection between $C_{\mathrm{NJ}}(\cdot, X)$ and the modulus of convexity $\delta_{X}$ is established. Finally, we investigate the constants $C_{\mathrm{NJ}}(\cdot, X)$ when $X$ is a u-space. This leads to an alternative proof of (iv).

Received 24th June, 2002

Supported by Thailand Research Fund under grant BRG/01/2544. The third author was also supported by the Royal Golden Jubilee program under grant PHD/0145/2542.

Copyright Clearance Centre, Inc. Serial-fee code: 0004-9727/03 \$A2.00+0.00. 


\section{Preliminaries}

Throughout the paper we let $X$ and $X^{*}$ stand for a Banach space and its dual space, respectively. By a non-trivial Banach space $X$ we shall mean that either $\mathrm{X}$ is a real space with $\operatorname{dim} X \geqslant 2$, or a complex space with $\operatorname{dim} X \geqslant 1$. We shall denote by $B_{X}$ and $S_{X}$ the closed unit ball and the unit sphere of $X$, respectively. For a sequence $\left(x_{n}\right)$ in $\mathrm{X}, x_{n} \stackrel{w}{\rightarrow} x$ stands for weak convergence to $x$. For $x \in X \backslash\{0\}$, let $\nabla_{x}$ denote the set of norm 1 supporting functionals at $x$. This is the subdifferential of the norm at the point $x$, which is nonempty by the Hanh-Banach Theorem.

We shall say that a nonempty weakly compact convex subset $C$ of $X$ has the fixed point property ( $f p p$ for short) if every nonexpansive mapping $T: C \rightarrow C$ has a fixed point (that is, there exists $x \in C$ such that $T(x)=x$ ). Recall that $T$ is nonexpansive if $\|T x-T y\| \leqslant\|x-y\|$ for every $x, y \in C$. We shall say that $X$ has the fixed point property $(f p p)$ if every weakly compact convex subset of $X$ has the $f p p$. Let $A$ be a nonempty bounded set in $X$. The number $r(A)=\inf \left\{\sup _{y \in A}\|x-y\|: x \in A\right\}$ is called the Chebyshev radius of $A$. The number $\operatorname{diam} A=\sup _{x, y \in A}\|x-y\|$ is called the diameter of $A$. A Banach space $X$ has normal structure if

$$
r(A)<\operatorname{diam} A
$$

for every bounded convex closed subset $A$ of $X$ with $\operatorname{diam} A>0$. When (2.1) holds for every weakly compact convex subset $A$ of $X$ with $\operatorname{diam} A>0$, we say $X$ has weak normal structure. Normal structure and weak normal structure coincide if $X$ is reflexive. A space $X$ is said to have uniform normal structure if $\inf \{(\operatorname{diam} A) /(r(A))\}>1$, where the infimum is taken over all bounded convex closed subsets $A$ of $X$ with $\operatorname{diam} A>0$. Weak normal structure, as well as many other properties imply the fixed point property. Some relevant papers are Opial [22], Kirk [16], Sims [24], Garcia-Falset [7], and Gacia-Falset and Sims [8].

The modulus of convexity of $X$ (see $[3,4,19,20,21])$ is the function $\delta_{X}:[0,2]$ $\rightarrow[0,1]$ defined by

$$
\delta_{X}(\varepsilon)=\inf \left\{1-\left\|\frac{x+y}{2}\right\|: x, y \in S_{X},\|x-y\| \geqslant \varepsilon\right\} .
$$

When $X$ is non-trivial, we can deduce that

$$
\begin{aligned}
\delta_{X}(\varepsilon) & =\inf \left\{1-\left\|\frac{x+y}{2}\right\|: x, y \in B_{X},\|x-y\| \geqslant \varepsilon\right\} \\
& =\inf \left\{1-\left\|\frac{x+y}{2}\right\|: x, y \in S_{X},\|x-y\|=\varepsilon\right\} \\
& =\inf \left\{1-\left\|\frac{x+y}{2}\right\|: x, y \in B_{X},\|x-y\|=\varepsilon\right\} .
\end{aligned}
$$


If $\delta_{X}(1)>0$, then $X$ has uniform normal structure (see [9]).

The modulus of smoothness of $X$ (see $[3,4,19,20]$ ) is the function $\rho_{X}:[0, \infty)$ $\rightarrow[0, \infty)$ defined by

$$
\begin{aligned}
\rho_{X}(\tau) & =\sup \left\{\frac{\|x+\tau y\|+\|x-\tau y\|}{2}-1: x, y \in S_{X}\right\} \\
& =\sup \left\{\frac{\tau \varepsilon}{2}-\delta_{X^{*}}(\varepsilon): \varepsilon \in[0,2]\right\} .
\end{aligned}
$$

A space $X$ is called uniformly convex if $\delta_{X}(\varepsilon)>0$ for all $0<\varepsilon<2$. It is called uniformly smooth if $\rho_{X}^{\prime}(0)=\lim _{\tau \rightarrow 0}\left(\rho_{X}(\tau)\right) / \tau=0$. Uniformly convex spaces and uniformly smooth spaces are examples of $u$-spaces, where a space $X$ is called a $u$-space if for any $\varepsilon>0$, there exists $\delta>0$ such that for each $x, y \in S_{X}$,

$$
\left\|\frac{x+y}{2}\right\|>1-\delta \Rightarrow f(y)>1-\varepsilon \text { for all } f \in \nabla_{x} .
$$

The notion of u-spaces was introduced by Lau [18]. Examples of uniformly convex spaces are the spaces $L^{p}(\Omega)$ where $\Omega$ is a measure space such that $L^{p}(\Omega)$ is at least two dimensional and $1<p<\infty$.

A Banach space $X$ is called uniformly nonsquare provided that there exists $\delta>0$ such that if $x, y \in S_{X}$, then $\|x+y\| / 2 \leqslant 1-\delta$ or $\|x-y\| / 2 \leqslant 1-\delta$. Uniformly nonsquare spaces are superreflexive (see James [11]). Every u-space is uniformly nonsquare (see Lau [18]), hence, it is superreflexive.

The Jordan-von Neumann constant $C_{\mathrm{NJ}}(X)$ of a Banach space $X$ is defined by

$$
\begin{aligned}
C_{\mathrm{NJ}}(X) & =\sup \left\{\frac{\|x+y\|^{2}+\|x-y\|^{2}}{2\left(\|x\|^{2}+\|y\|^{2}\right)}: x, y \in X \text { not both zero }\right\} \\
& =\sup \left\{\frac{\|x+y\|^{2}+\|x-y\|^{2}}{2\left(\|x\|^{2}+\|y\|^{2}\right)}: x \in S_{X}, y \in B_{X}\right\} .
\end{aligned}
$$

REMARK 2.1. We collect together some properties of the Jordan-von Neumann constant $C_{\mathrm{NJ}}(X)$ (see $\left.[2,12,13,14,15,25]\right)$ :

(1) $1 \leqslant C_{\mathrm{NJ}}(X) \leqslant 2$.

(2) $X$ is a Hilbert space if and only if $C_{\mathrm{NJ}}(X)=1$.

(3) $C_{\mathrm{NJ}}(X)=C_{\mathrm{NJ}}\left(X^{*}\right)$.

(4) $X$ is uniformly nonsquare if and only if $C_{\mathrm{NJ}}(X)<2$ and this happens if and only if $\delta_{X}(\varepsilon)>0$ for some $\varepsilon \in(0,2)$.

(5) If $C_{\mathrm{NJ}}(X)<5 / 4$ then $X$, as well as its dual $X^{*}$, have uniform normal structure, and hence both $X$ and $X^{*}$ have the fixed point property. 
One technique used in this paper is the "ultraproduct" technique. We refer to Askoy and Khamsi [1] and Sims [23] for a complete discussion on the topic. However, let us briefly recall the construction of an ultrapower of a Banach space $X$. As a first step we consider the space $l_{\infty}(X)$ consisting of all bounded sequences $\left(x_{n}\right)$ of elements of $X$. The norm in $l_{\infty}(X)$ is given by the formula $\left\|\left(x_{n}\right)\right\|=\sup _{n \in \mathbb{N}}\left\|x_{n}\right\|$, where $\mathbb{N}$ is the set of positive integers. Now, let $\mathcal{U}$ be an ultrafilter on $\mathbb{N}$. The set $\mathcal{N}=\left\{\left(x_{n}\right) \in l_{\infty}(X): \lim _{\mathcal{U}}\left\|x_{n}\right\|=0\right\}$ is a closed linear subspace of $l_{\infty}(X)$. Here, $\lim _{\mathcal{U}}$ stands for the limit over the ultrafilter $\mathcal{U}$. The ultrapower $\tilde{X}$ of $X$ with respect to $\mathcal{U}$ is defined to be the quotient space $l_{\infty}(X) / \mathcal{N}$. By $\tilde{x}$ we denote the equivalent class of $x=\left(x_{n}\right)$. From the definition of the quotient norm, we can derive the following canonical formula $\|\tilde{x}\|=\lim _{\mathcal{U}}\left\|x_{n}\right\|$. Identifying an element $x \in X$ with the equivalence class of the constant sequence $(x, x, \ldots)$, we can treat $X$ as a subspace of $\tilde{X}$. In what follow, we shall consider only non-trivial ultrafilters on the set of positive integers. Under this setting, the ultrapower $\tilde{X}$ is finitely representable in $X$. Consequently, $\tilde{X}$ inherits all finite-dimensional geometrical properties of $X$.

Definition 2.2: Let $\mathcal{P}$ be a Banach space property. We say that a Banach space $X$ has the property super- $\mathcal{P}$ if every Banach space finitely representable in $X$ has property $\mathcal{P}$.

Theorem 2.3. (See [1, Theorem 3.5].) Let $X$ and $Y$ be Banach spaces and suppose that $Y$ is finitely representable in $X$. Then there is an ultrafilter $\mathcal{U}$ on the set $\mathbb{N}$ such that $Y$ is isometrically isomorphic to a subspace of $\widetilde{X}$.

We remark that when the property $\mathcal{P}$ is hereditary: that is, any subspace of a space with $\mathcal{P}$ also has $\mathcal{P}$, one has the following stronger conclusion.

Corollary 2.4. (See [1].) Let $\mathcal{P}$ be a Banach space property which is inherited by subspaces. Then a Banach space $X$ has super- $\mathcal{P}$ if and only if every ultrapower $\tilde{X}$ of $X$ has $\mathcal{P}$.

THEOREM 2.5. (See [1].) Let $X$ be a Banach space. If $X$ has super-normal structure, then $X$ has uniform normal structure.

\section{RESUlts}

Let us begin with our generalisation of the Jordan-von Neumann constant. For $a \geqslant 0$ define,

$$
\begin{gathered}
C_{\mathrm{NJ}}(a, X)=\sup \left\{\frac{\|x+y\|^{2}+\|x-z\|^{2}}{2\|x\|^{2}+\|y\|^{2}+\|z\|^{2}}: x, y, z \in X\right. \text { not all zero } \\
\text { and }\|y-z\| \leqslant a\|x\|\}
\end{gathered}
$$




$$
\begin{aligned}
& =\sup \left\{\frac{\|x+y\|^{2}+\|x-z\|^{2}}{2\|x\|^{2}+\|y\|^{2}+\|z\|^{2}}: x, y, z \in B_{X}\right. \text { not all zero } \\
& \quad \text { and }\|y-z\| \leqslant a\|x\|\} \\
& =\sup \left\{\frac{\|x+y\|^{2}+\|x-z\|^{2}}{2\|x\|^{2}+\|y\|^{2}+\|z\|^{2}}: x, y, z \in B_{X}\right. \text { of which at least one } \\
& \text { belongs to } \left.S_{X} \text { and }\|y-z\| \leqslant a\|x\|\right\} .
\end{aligned}
$$

REMARK 3.1.

(1) Obviously, $C_{\mathrm{NJ}}(0, X)=C_{\mathrm{NJ}}(X)$ (see (2.5)).

(2) $C_{\mathrm{NJ}}(a, X)$ is a nondecreasing function with respect to $a$.

(3) If $C_{\mathrm{NJ}}(a, X)<2$, for some $a \geqslant 0$, then $C_{\mathrm{NJ}}(X)<2$ and consequently $X$ is uniformly nonsquare (see Remark 2.1(4)).

(4) $1+\left(4 a / 4+a^{2}\right) \leqslant C_{\mathrm{NJ}}(a, X) \leqslant 2$ for all $a \geqslant 0$ and $C_{\mathrm{NJ}}(a, X)=2$ for all $a \geqslant 2$.

To see that (4) is true, we begin by proving the left inequality. For this, we take any $x \in S_{X}$ and put $y=(a / 2) x=-z$. We then have $y-z=a x$ and so,

$$
\begin{aligned}
C_{\mathrm{N} J}(a, X) & \geqslant \frac{\|x+y\|^{2}+\|x-z\|^{2}}{2\|x\|^{2}+\|y\|^{2}+\|z\|^{2}}=\frac{(1+(a / 2))^{2}\|x\|^{2}+(1+(a / 2))^{2}\|x\|^{2}}{2\|x\|^{2}+2\left(a^{2} / 4\right)\|x\|^{2}} \\
& =\frac{2(1+(a / 2))^{2}}{2\left(1+\left(a^{2} / 4\right)\right)}=\frac{4+4 a+a^{2}}{4+a^{2}}=1+\frac{4 a}{4+a^{2}} .
\end{aligned}
$$

Next, we show that $C_{\mathrm{NJ}}(a, X) \leqslant 2$. By the triangle inequality, we have

$$
\begin{aligned}
\|x+y\|^{2}+\|x-z\|^{2} & \leqslant\left(\|x\|^{2}+2\|x\|\|y\|+\|y\|^{2}\right)+\left(\|x\|^{2}+2\|x\|\|z\|+\|z\|^{2}\right) \\
& \leqslant\left(2\|x\|^{2}+2\|y\|^{2}\right)+\left(2\|x\|^{2}+2\|z\|^{2}\right) \\
& =4\|x\|^{2}+2\|y\|^{2}+2\|z\|^{2}
\end{aligned}
$$

from which it is clear that $C_{\mathrm{NJ}}(a, X) \leqslant 2$. Finally, we observe that the function $a \mapsto 1+\left(4 a / 4+a^{2}\right)$ is strictly increasing on $[0,2]$ and attains its maximum of 2 at $a=2$. It follows that $C_{\mathrm{NJ}}(a, X)=2$ for all $a \geqslant 2$.

EXAMPLES 3.2. (1) $\left(l_{\infty}-l_{1}\right.$ norm) Let $X=\mathbb{R}^{2}$ be equipped with the norm defined by

$$
\|x\|= \begin{cases}\|x\|_{\infty} & \text { if } x_{1} x_{2} \geqslant 0 \\ \|x\|_{1} & \text { if } x_{1} x_{2} \leqslant 0\end{cases}
$$

Take $x=(1,1), y=(0,1)$ and $z=(-1,0)$. Then we have $y-z=(1,1)=x$ and $\|x+y\|=\|(1,2)\|_{\infty}=2,\|x-z\|=\|(2,1)\|_{\infty}=2,\|z\|=1$. So $2=(4+4) / 4$ 
$=\left(\|x+y\|^{2}+\|x-z\|^{2}\right) /\left(2\|x\|^{2}+\|y\|^{2}+\|z\|^{2}\right) \leqslant C_{\mathrm{NJ}}(1, X) \leqslant 2$. Hence $C_{\mathrm{NJ}}(1, X)$ $=2$. It is not difficult to see that $\delta_{X}(\varepsilon)=\max \{0,(\varepsilon-1) / 2\}$ and so $\delta_{X}(1)=0$. We shall shortly see (Remark 3.12(1)) that this implies $C_{\mathrm{NJ}}(0, X) \geqslant 5 / 4$, however, we do not know its exact value. This example shows that sometimes it is easy to compute $C_{\mathrm{NJ}}(a, X)$ at some point $a \in(0,2)$, but not at $a=0$.

(2) Let $1<p<2$ and let the norm on $X=\mathbb{R}^{2}$ now be defined by

$$
\|x\|= \begin{cases}\|x\|_{1} & \text { if } x_{1} x_{2} \geqslant 0 \\ \|x\|_{p} & \text { if } x_{1} x_{2} \leqslant 0\end{cases}
$$

Under this norm, it can be shown that $\delta_{X}(1)=0, C_{N J}(X)=1+2^{2 / p-2}, J(X)$ $\geqslant 2^{1 / p}$ and $C_{\mathrm{NJ}}(1, X)<2$, where James' nonsquare constant $J(X)$ is defined by $J(X)$ $=\sup \left\{\min \{\|x+y\|, \| x-y\}: x, y \in S_{X}\right\}$. The verification that $C_{\mathrm{NJ}}(1, X)<2$ follows by an argument similar to that given later in the proof of Theorem 3.15. We shall shortly see that all spaces $X$ with $C_{\mathrm{NJ}}(1, X)<2$ have uniform normal structure (Corollary 3.7). This example also reveals that we may have $C_{\mathrm{NJ}}(X)$ close to 2 but still have uniform normal structure (also see the observation given later at the beginning of Remark 3.16).

These examples show that information on $C_{\mathrm{NJ}}(a, X)$ for general $a$ proves to be useful. We note in passing that $C_{\mathrm{NJ}}\left(1, l_{2}(X)\right)<2$ whenever $C_{\mathrm{NJ}}(1, X)<2$, where $l_{2}(X)$ is the space of sequences $\left(x_{n}\right)$ of elements of $X$ for which the sequence of norms $\left(\left\|x_{n}\right\|\right)$ is in $l_{2}$, with the norm of $\left(x_{n}\right)$ defined to be the $l_{2}$-norm of $\left(\left\|x_{n}\right\|\right)$.

We aim to show that the generalised Jordan-von Neumann constants $C_{\mathrm{NJ}}(a, X)$ of the space $X$ and $C_{\mathrm{NJ}}(a, \tilde{X})$ of its ultrapower coincide. Before that we need to establish the continuity of the function $C_{\mathrm{NJ}}(\cdot, X)$.

Proposition 3.3. $C_{\mathrm{NJ}}(\cdot, X)$ is a continuous function on $[0, \infty)$.

Proof: We have already noted that $C_{\mathrm{NJ}}(\cdot, X)$ is nondecreasing, thus suppose that for some $a>0$,

$$
\sup _{b<a} C_{\mathrm{NJ}}(b, X)=\alpha<\beta<\gamma=\inf _{b>a} C_{\mathrm{NJ}}(b, X) .
$$

Choose $\gamma_{n} \downarrow a$ and $x_{n}, y_{n}, z_{n} \in B_{X}$ of which at least one belongs to $S_{X}$ and such that $\left\|y_{n}-z_{n}\right\|=\gamma_{n}\left\|x_{n}\right\|$ and $g\left(x_{n}, y_{n}, z_{n}\right) \geqslant \beta$ for all $n \in \mathbb{N}$. Here $g(x, y, z)$ $=\left(\|x+y\|^{2}+\|x-z\|^{2}\right) /\left(2\|x\|^{2}+\|y\|^{2}+\|z\|^{2}\right)$. Choose $\eta_{n} \downarrow 1$ such that $\gamma_{n} / \eta_{n}<a$ for all $n$. Thus, $g\left(\eta_{n} x_{n}, y_{n}, z_{n}\right)=g\left(x_{n},\left(y_{n} / \eta_{n}\right),\left(z_{n} / \eta_{n}\right)\right) \leqslant \alpha$ for all $n \in \mathbb{N}$. Take a subsequence $\left(n^{\prime}\right)$ of $(n)$ such that all the sequences

$$
\left\|x_{n^{\prime}}+y_{n^{\prime}}\right\|,\left\|x_{n^{\prime}}-z_{n^{\prime}}\right\|,\left\|x_{n^{\prime}}\right\|,\left\|y_{n^{\prime}}\right\| \text { and }\left\|z_{n^{\prime}}\right\|
$$

converge. As $\left\|x_{n}+w\right\|-\left(\eta_{n}-1\right)\left\|x_{n}\right\| \leqslant\left\|\eta_{n} x_{n}+w\right\| \leqslant\left\|x_{n}+w\right\|+\left(\eta_{n}-1\right)\left\|x_{n}\right\|$ for any $w \in X$ and $\eta_{n} \rightarrow 1$, we have $\lim _{n^{\prime}}\left\|\eta_{n^{\prime}} x_{n^{\prime}}+y_{n^{\prime}}\right\|=\lim _{n^{\prime}}\left\|x_{n^{\prime}}+y_{n^{\prime}}\right\|$ and $\lim _{n^{\prime}} \| \eta_{n^{\prime}} x_{n^{\prime}}$ 
$-z_{n^{\prime}}\left\|=\lim _{n^{\prime}}\right\| x_{n^{\prime}}-z_{n^{\prime}} \|$. Consequently, $\beta-\alpha \leqslant g\left(x_{n^{\prime}}, y_{n^{\prime}}, z_{n^{\prime}}\right)-g\left(\eta_{n^{\prime}} x_{n^{\prime}}, y_{n^{\prime}}, z_{n^{\prime}}\right)$ $\rightarrow 0$, a contradiction. This finishes the proof when $a>0$.

For $a=0$, given $\varepsilon>0$ we take a triple $\left(x_{n}, y_{n}, z_{n}\right)$ in $B_{X}^{3}$ with at least one of $x_{n}, y_{n}, z_{n}$ belonging to $S_{X},\left\|y_{n}-z_{n}\right\|=\alpha_{n}\left\|x_{n}\right\|, \alpha_{n} \downarrow 0$, and

$$
C_{\mathrm{NJ}}(0+, X)-\varepsilon:=\inf _{a>0} C_{\mathrm{NJ}}(a, X)-\varepsilon<\lim _{n \rightarrow \infty} g\left(x_{n}, y_{n}, z_{n}\right) .
$$

Put $\varepsilon_{n}=4 \alpha_{n}+\alpha_{n}^{2}$ and $\gamma_{n}=\alpha_{n}\left\|x_{n}\right\|\left(\left\|y_{n}\right\|-\alpha_{n}\left\|x_{n}\right\|\right)$. Thus $\varepsilon_{n}, \gamma_{n} \rightarrow 0$. Passing through subsequences if necessary, we may assume that $\lim _{n \rightarrow \infty}\left(\left\|x_{n}\right\|^{2}+\left\|y_{n}\right\|^{2}\right)=b$ exists. By the choice of $\left(x_{n}, y_{n}, z_{n}\right)$ we see that $b \neq 0$. Next we observe that, for all large $n$,

$$
\begin{aligned}
g\left(x_{n}, y_{n}, z_{n}\right) & \leqslant \frac{\left\|x_{n}+y_{n}\right\|^{2}+\left\|x_{n}-y_{n}\right\|^{2}+\varepsilon_{n}}{2\left\|x_{n}\right\|^{2}+2\left\|y_{n}\right\|^{2}-\gamma_{n}} \\
& \leqslant g\left(x_{n}, y_{n}, y_{n}\right)+\frac{\varepsilon_{n}+\gamma_{n} g\left(x_{n}, y_{n}, y_{n}\right)}{2\left\|x_{n}\right\|^{2}+2\left\|y_{n}\right\|^{2}-\gamma_{n}} \\
& \leqslant C_{\mathrm{NJ}}(X)+\frac{\varepsilon_{n}+\gamma_{n} C_{\mathrm{NJ}}(X)}{2\left\|x_{n}\right\|^{2}+2\left\|y_{n}\right\|^{2}-\gamma_{n}} .
\end{aligned}
$$

Thus $C_{\mathrm{NJ}}(0+, X)-\varepsilon<C_{\mathrm{NJ}}(X) \leqslant C_{\mathrm{NJ}}(0+, X)$ for all $\varepsilon>0$. Therefore $C_{\mathrm{NJ}}(0+, X)$ $=C_{\mathrm{NJ}}(X)$ which implies that $C_{\mathrm{NJ}}(\cdot, X)$ is continuous at 0 . Hence the continuity of $C_{\mathrm{NJ}}(\cdot, X)$ is established.

We are now ready to obtain an important tool.

Corollary 3.4. $C_{\mathrm{NJ}}(a, X)=C_{\mathrm{NJ}}(a, \tilde{X})$.

Proof: Clearly, $C_{\mathrm{NJ}}(a, X) \leqslant C_{\mathrm{NJ}}(a, \tilde{X})$. To show $C_{\mathrm{NJ}}(a, X) \geqslant C_{\mathrm{NJ}}(a, \tilde{X})$, let $\delta>0, \alpha \in[0, a]$ and suppose $\tilde{x}, \tilde{y}, \tilde{z} \in \tilde{X}$ not all of which are zero and for which $\|\tilde{y}-\tilde{z}\|=\alpha\|\tilde{x}\|$. If $\tilde{x}=0$, then $g(\tilde{x}, \tilde{y}, \tilde{z})=1 \leqslant C_{\mathrm{NJ}}(a, X)$. If $\tilde{x} \neq 0$, choose $\varepsilon>0$ such that $\varepsilon<\delta\|\widetilde{x}\|$. Since

$$
c:=\frac{\|\tilde{x}+\tilde{y}\|^{2}+\|\tilde{x}-\tilde{z}\|^{2}}{2\|\tilde{x}\|^{2}+\|\tilde{y}\|^{2}+\|\tilde{z}\|^{2}}=\lim _{\mathcal{U}} \frac{\left\|x_{n}+y_{n}\right\|^{2}+\left\|x_{n}-z_{n}\right\|^{2}}{2\left\|x_{n}\right\|^{2}+\left\|y_{n}\right\|^{2}+\left\|z_{n}\right\|^{2}}:=\lim _{\mathcal{U}} c_{n},
$$

the set $\left\{n \in \mathbb{N}:\left|c_{n}-c\right|<\delta\right.$ and $\left.\left\|y_{n}-z_{n}\right\| \leqslant \alpha\left\|x_{n}\right\|+\varepsilon<(\alpha+\delta)\left\|x_{n}\right\|\right\}$ belongs to $\mathcal{U}$. In particular,

$$
\begin{aligned}
c & <g\left(\dot{x_{n}}, y_{n}, z_{n}\right)+\delta \\
& \leqslant C_{\mathrm{NJ}}(a+\delta, X)+\delta \quad \text { for some } n .
\end{aligned}
$$

The inequality $C_{\mathrm{NJ}}(a, \tilde{X}) \leqslant C_{\mathrm{NJ}}(a, X)$ follows from the arbitrariness of $\delta$ and the continuity of $C_{\mathrm{NJ}}(\cdot, X)$. 
This result also follows from the fact that the parameterised Jordan-von Neumann constant is finitely determined.

The following Lemma is a modification of [6, Lemma 2.3].

LEMMA 3.5. Let $X$ be a Banach space without weak normal structure, then for any $0<\varepsilon<1$ and each $1 / 2<r \leqslant 1$, there exist $x_{1} \in S_{X}$ and $x_{2}, x_{3} \in r S_{X}$ satisfying

(i) $x_{2}-x_{3}=a x_{1}$ with $|a-r|<\varepsilon$,

(ii) $\left\|x_{1}-x_{2}\right\|>1-\varepsilon$, and

(iii) $\left\|x_{1}+x_{2}\right\|>(1+r)-\varepsilon,\left\|x_{3}+\left(-x_{1}\right)\right\|>(3 r-1)-\varepsilon$.

Proof: Put $\eta=\min \{(\varepsilon / 12 r), 2-(1 / r)\}$, and let $z_{n}$ be a sequence in $S_{X}$ with $z_{n} \stackrel{w}{\rightarrow} 0$ and

$$
1-\eta<\left\|z_{n+1}-z\right\|<1+\eta
$$

for sufficiently large $n$ and for any $z \in \operatorname{co}\left\{z_{k}\right\}_{k=1}^{n}$. Take $n_{0} \in \mathbb{N}, y \in \operatorname{co}\left\{z_{n}\right\}_{n=1}^{n_{0}}$ and a norm 1 supporting functional $f$ of $z_{1}$ such that

$$
\|y\|<\eta,\left|\left\langle f, z_{n_{0}}\right\rangle\right|<\eta, 1-\eta<\left\|z_{n_{0}}-z_{1}\right\|,\left\|z_{n_{0}}-\frac{z_{1}}{2}\right\|<1+\eta
$$

and

$$
\left\|\frac{z_{1}-z_{n_{0}}}{\left\|z_{1}-z_{n_{0}}\right\|}-z_{n_{0}}\right\|>2-3 \eta
$$

Put $x_{1}=\left(z_{1}-z_{n_{0}}\right) /\left(\left\|z_{1}-z_{n_{0}}\right\|\right), x_{2}=r z_{1}$ and $x_{3}=r z_{n_{0}}$. We show that (i), (ii) and (iii) hold. We first note that $x_{2}-x_{3}=r\left(z_{1}-z_{n_{0}}\right)=r\left\|z_{1}-z_{n_{0}}\right\| x_{1}$. Observe that $1-\eta<\left\|z_{1}-z_{n_{0}}\right\|<1+\eta$, so $\left|r\left\|z_{1}-z_{n_{0}}\right\|-r\right|<r \eta<\varepsilon$, hence (i) holds. Next, since $1 / 2<r \leqslant 1$,

$$
\left|r\left(1+\left\|z_{1}-z_{n_{0}}\right\|\right)-1\right|=r\left(1+\left\|z_{1}-z_{n_{0}}\right\|\right)-1<r(2+\eta)-1=(2 r-1)+r \eta
$$

This implies

$$
\begin{aligned}
\left\|x_{1}-x_{2}\right\| & =\left\|r x_{1}+(1-r) x_{1}-r\right\| z_{1}-z_{n_{0}}\left\|x_{1}-r z_{n_{0}}\right\| \\
& \geqslant r\left\|x_{1}-z_{n_{0}}\right\|-\left|1-r-r\left\|z_{1}-z_{n_{0}}\right\|\right| \\
& >r(2-3 \eta)-(2 r-1)-r \eta \\
& =2 r-3 r \eta-2 r+1-r \eta \\
& >1-\varepsilon .
\end{aligned}
$$

Thus (ii) follows. 
To verify (iii) we first note the estimate $\left\|r z_{1}-r z_{n_{0}}-x_{1}\right\|=\|(1-r) x_{1}+r\left(x_{1}\right.$ $\left.-\left(z_{1}-z_{n_{0}}\right)\right) \| \leqslant(1-r)+r \eta<(1-r)+r \eta$. Using this we have,

$$
\begin{aligned}
\left\|x_{1}-x_{3}\right\| & =\left\|x_{1}-r z_{n_{0}}\right\| \\
& \geqslant\left\|r z_{n_{0}}-\left(r z_{1}-r z_{n_{0}}\right)\right\|-\left\|r z_{1}-r z_{n_{0}}-x_{1}\right\| \\
& \geqslant 2 r\left\|z_{n_{0}}-\frac{z_{1}}{2}\right\|-(1-r)-r \eta \\
& >2 r-2 r \eta-(1-r)-r \eta \\
& >(3 r-1)-\varepsilon .
\end{aligned}
$$

We now estimate $\left\|x_{1}+x_{2}\right\|$. From the definition of $f$, we have

$$
\begin{aligned}
\left\|x_{1}+x_{2}\right\| & \geqslant\left\langle f, x_{1}+r z_{1}\right\rangle=r+\left\langle f, x_{1}\right\rangle \\
& =r+\frac{\left\langle f, z_{1}\right\rangle-\left\langle f, z_{n_{0}}\right\rangle}{\left\|z_{1}-z_{n_{0}}\right\|} \\
& >r+\frac{1-\eta}{1+\eta} \\
& =(r+1)-\frac{2 \eta}{1+\eta} \\
& >(r+1)-\varepsilon .
\end{aligned}
$$

The proof of the Lemma is now complete.

We now obtain sufficient conditions for $X$ to have uniform normal structure, the second of which improves [13, Corollary 4] which states that "A Banach space $X$ with $C_{\mathrm{NJ}}(X)<5 / 4$ has uniform normal structure."

THEOREM 3.6. Let $X$ be a Banach space. If

$$
C_{\mathrm{NJ}}(r, X)<\frac{(1+r)^{2}+(3 r-1)^{2}}{2\left(1+r^{2}\right)}, \quad \text { for some } r \in\left(\frac{1}{2}, 1\right]
$$

or

$$
C_{\mathrm{NJ}}(0, X)<\frac{3+\sqrt{5}}{4}
$$

then $X$ has uniform normal structure.

ProOF: It suffices to show that these conditions imply $X$ has normal structure. As then, by Corollary 3.4 , it follows that $\tilde{X}$ also has normal structure, so $X$ has super-normal structure, by Corollary 2.4, and hence $X$ has uniform normal structure by Theorem 2.5 . 
For the case $C_{\mathrm{NJ}}(r, X)<\left((1+r)^{2}+(3 r-1)^{2}\right) /\left(2\left(1+r^{2}\right)\right)$ we first observe that from Remark 3.1(3), $X$ is uniformly nonsquare and so in turn is reflexive. Thus, normal structure and weak normal structure coincide. It then suffices to prove that $X$ has weak normal structure.

By the continuity of $C_{\mathrm{NJ}}(\cdot, X), C_{\mathrm{NJ}}\left(r^{\prime}, X\right)<\left((1+r)^{2}+(3 r-1)^{2}\right) /\left(2\left(1+r^{2}\right)\right)$ for some $r^{\prime}>r$. Choose $m \in \mathbb{N}$ such that $r+(1 / m) \leqslant r^{\prime}$. Suppose $X$ does not have weak normal structure. By Lemma 3.5 there exist $x_{n} \in S_{X}$ and $y_{n}, z_{n} \in r S_{X}$ such that, for each $n \in \mathbb{N}$,

$$
\begin{aligned}
y_{n}-z_{n}=\alpha_{n} x_{n} \text { with }\left|\alpha_{n}-r\right|<\frac{1}{n+m} \\
\left\|x_{n}-y_{n}\right\|^{2}>\left(1-\frac{1}{n+m}\right)^{2},\left\|x_{n}+y_{n}\right\|^{2}>\left(1+r-\frac{1}{n+m}\right)^{2},
\end{aligned}
$$

and

$$
\left\|x_{n}-z_{n}\right\|^{2}>\left((3 r-1)-\frac{1}{n+m}\right)^{2} .
$$

Observe that $\left\|y_{n}-z_{n}\right\|=\alpha_{n}<r+(1 / n+m)<r+(1 / m) \leqslant r^{\prime}$ and

$$
\liminf _{n \rightarrow \infty}\left\|x_{n}+y_{n}\right\|^{2} \geqslant(1+r)^{2} \text { and } \liminf _{n \rightarrow \infty}\left\|x_{n}-z_{n}\right\|^{2} \geqslant(3 r-1)^{2} \text {. }
$$

Thus

$$
\begin{aligned}
\frac{(1+r)^{2}+(3 r-1)^{2}}{2\left(1+r^{2}\right)} & \leqslant \liminf _{n \rightarrow \infty} \frac{\left\|x_{n}+y_{n}\right\|^{2}+\left\|x_{n}-z_{n}\right\|^{2}}{2\left\|x_{n}\right\|^{2}+\left\|y_{n}\right\|^{2}+\left\|z_{n}\right\|^{2}} \\
& \leqslant C_{\mathrm{NJ}}\left(r^{\prime}, X\right) \\
& <\frac{(1+r)^{2}+(3 r-1)^{2}}{2\left(1+r^{2}\right)} .
\end{aligned}
$$

This contradiction shows that $X$ must have weak normal structure as desired.

For the case $C_{\mathrm{NJ}}(0, X)<(3+\sqrt{5}) / 4$, we first show that $C_{\mathrm{NJ}}(0, X)$ $<\left((1+r)^{2}+1\right) /\left(2\left(1+r^{2}\right)\right)$ for any $r \in(1 / 2,1]$. The proof of this is the same as above except that here we consider the lower bound $(1-(1 / m+n))^{2}$ for $\left\|x_{n}-y_{n}\right\|^{2}$ instead of the one for $\left\|x_{n}-z_{n}\right\|^{2}$. Thus (3.1) becomes

$$
\frac{(1+r)^{2}+1}{2\left(1+r^{2}\right)} \leqslant \liminf _{n \rightarrow \infty} \frac{\left\|x_{n}+y_{n}\right\|^{2}+\left\|x_{n}-y_{n}\right\|^{2}}{2\left(\left\|x_{n}\right\|^{2}+\left\|y_{n}\right\|^{2}\right)} \leqslant C_{\mathrm{NJ}}(0, X)<\frac{(1+r)^{2}+1}{2\left(1+r^{2}\right)}
$$

which is impossible. The conclusion now follows by noting that $\left((1+r)^{2}+1\right) /\left(2\left(1+r^{2}\right)\right)$ achieves a maximum of $(3+\sqrt{5}) / 4$ at $r=(\sqrt{5}-1) / 2 \in(1 / 2,1]$. 
Note. The restriction $r \in(1 / 2,1]$ in the first inequality of Theorem 3.6 reflects the fact that for $r \leqslant 1 / 2$ the right hand side is less than or equal to one. Indeed, from Remark 3.1(4) the first inequality in Theorem 3.6 is only possible if

$$
\frac{(1+r)^{2}+(3 r-1)^{2}}{2\left(1+r^{2}\right)} \geqslant 1+\frac{4 r}{4+r^{2}},
$$

that is, if $r \in\left(r_{1}, 1\right]$ where $r_{1} \doteq 0.87$ is the real root of the polynomial $2 x^{3}-3 x^{2}+8 x-6$. Thus, Theorem 3.6 only gives us information near $r=1$.

Corollary 3.7. Let $X$ be a Banach space. If $C_{\mathrm{NJ}}(1, X)<2$, then $X$ has uniform normal structure.

Proof: This follows immediately from Theorem 3.6 with $r=1$.

Utilising Corollary 3.7, Tasena [26] has shown " $C_{\mathrm{NJ}}(a, X)<(1+a)^{2} /\left(1+a^{2}\right)$ for some $a \in(0,1]$ implies $X$ has uniform normal structure". This improvement of Theorem 3.6 is quite strong since

$$
\frac{(1+a)^{2}}{1+a^{2}}>\max \left(1+\frac{4 a}{4+a^{2}} \frac{(1+a)^{2}+(3 a-1)^{2}}{2\left(1+a^{2}\right)}\right) \text { for } a \in(0,1) .
$$

We now consider the case when $X$ is a Hilbert space, thereby extending Remark 2.1(2).

THEOREM 3.8. Let $H$ be a Hilbert space. Then

$$
C_{\mathrm{NJ}}(a, H)=1+\frac{4 a}{4+a^{2}}
$$

for all $a \in[0,2]$.

Proof: Let $a \in[0,2]$ and $x, y, z \in H$ with $x \neq 0$ and $\|y-z\|=\alpha\|x\|$ for some $\alpha \in[0, a]$. Then

$$
\begin{aligned}
\frac{\|x+y\|^{2}+\|x-z\|^{2}}{2\|x\|^{2}+\|y\|^{2}+\|z\|^{2}} & \leqslant \frac{2\|x\|^{2}+\|y\|^{2}+\|z\|^{2}+2\|x\|\|y-z\|}{2\|x\|^{2}+\|y\|^{2}+\|z\|^{2}} \\
& \leqslant 1+\frac{2 \alpha\|x\|^{2}}{2\|x\|^{2}+\left(\|y-z\|^{2}+\|y+z\|^{2}\right) / 2} \\
& \leqslant 1+\frac{2 \alpha\|x\|^{2}}{2\|x\|^{2}+\|y-z\|^{2} / 2} \\
& =1+\frac{4 \alpha}{4+\alpha^{2}} \\
& \leqslant 1+\frac{4 a}{4+a^{2}} .
\end{aligned}
$$

Thus, by Remark 3.1(4), $C_{\mathrm{NJ}}(a, H)=1+(4 a) /\left(4+a^{2}\right)$. 
Question. Is $X$ a Hilbert space if $C_{\mathrm{NJ}}(a, X)=1+(4 a) /\left(4+a^{2}\right)$ for some $a \in(0,2)$ ?

Theorem 3.8 and Corollary 3.7 give us the following

COROLlary 3.9. Every Hilbert space has uniform normal structure.

We now give a connection between the constant $C_{\mathrm{NJ}}(\cdot, X)$ and the modulus of convexity $\delta_{X}(\cdot)$ (see (2.2)).

THEOREM 3.10. Let $X$ be a Banach space, $\varepsilon \in[0,2]$, and $\beta \geqslant 0$. If $C_{\mathrm{NJ}}(\beta, X)<\left(4+(\varepsilon-\beta)^{2}\right) /\left(3+(\beta+1)^{2}\right)$, then $\delta_{X}(\varepsilon)>0$.

Proof: Suppose $\delta_{X}(\varepsilon)=0$, then there exist $x_{n}, y_{n} \in S_{X}$ such that $\left\|x_{n}-y_{n}\right\|=\varepsilon$ for all $n \in \mathbb{N}$ and $\lim _{n \rightarrow \infty}\left\|x_{n}+y_{n}\right\|=2$. Put $z_{n}=y_{n}-\beta x_{n}$. Then, for each $n \in \mathbb{N}$, we have $y_{n}-z_{n}=\beta x_{n},\left\|z_{n}\right\|=\left\|y_{n}-\beta x_{n}\right\| \leqslant 1+\beta$ and $\left\|x_{n}-z_{n}\right\| \geqslant\left|\left\|x_{n}-y_{n}\right\|-\left\|\beta x_{n}\right\|\right|$ $=|\varepsilon-\beta|$. Thus

$$
\frac{4+(\varepsilon-\beta)^{2}}{3+(\beta+1)^{2}} \leqslant \liminf _{n \rightarrow \infty} \frac{\left\|x_{n}+y_{n}\right\|^{2}+\left\|x_{n}-z_{n}\right\|^{2}}{2\left\|x_{n}\right\|^{2}+\left\|y_{n}\right\|^{2}+\left\|z_{n}\right\|^{2}} \leqslant C_{\mathrm{NJ}}(\beta, X)<\frac{4+(\varepsilon-\beta)^{2}}{3+(\beta+1)^{2}},
$$

a contradiction.

Note that Theorem 3.10 is applicable for all $\beta \in\left[0, \beta_{1}\right]$ where $\beta_{1}$ is the root of the equation

$$
1+\frac{4 \beta}{4+\beta^{2}}=\frac{4+(\varepsilon-\beta)^{2}}{3+(1+\beta)^{2}} .
$$

The above theorem immediately yields the following.

Corollary 3.11. If, for $\varepsilon \in[0,2], C_{\mathrm{NJ}}(0, X)<\left(4+\varepsilon^{2}\right) / 4$, then $\delta_{X}(\varepsilon)>0$. In particular, every Hilbert space is uniformly convex, that is, $\delta_{X}(\varepsilon)>0$ for every $\varepsilon \in(0,2)$.

\section{REMARK 3.12.}

(1) Corollary 3.11 shows that if $C_{\mathrm{NJ}}(X)<5 / 4$, then $\delta_{X}(1)>0$.

(2) $C_{\mathrm{NJ}}(0, X)<2$ if and only if $C_{\mathrm{NJ}}(0, X)<\left(4+\varepsilon^{2}\right) / 4$ for some $\varepsilon \in(0,2)$. Thus, this gives us a simpler proof of $[\mathbf{1 3}$, Theorem 1] which states that " $C_{\mathrm{NJ}}(0, X)<2$ if and only if $X$ is uniformly nonsquare."

(3) Since $C_{\mathrm{NJ}}(0, X)=C_{\mathrm{NJ}}\left(0, X^{*}\right)$, the corresponding results in Theorem 3.6 and Corollary 3.11 hold for $X^{*}$ as well.

Question. Does the equality $C_{\mathrm{NJ}}(a, X)=C_{\mathrm{NJ}}\left(a, X^{*}\right)$ hold for $a \in(0,2]$ ?

Corollary 3.13. If $C_{\mathrm{NJ}}(\cdot, X)$ is concave and $C_{\mathrm{NJ}}(a, X)<(3+\sqrt{5}$ $+(5-\sqrt{5}) a) / 4$ for some $a \in[0,1]$, then $X$ has uniform normal structure.

Proof: If $C_{\mathrm{NJ}}(1, X)<2$, we are done by Corollary 3.7. Let $C_{\mathrm{NJ}}(1, X)=2$ and suppose that $X$ does not have uniform normal structure. Therefore $C_{\mathrm{NJ}}(0, X)$ 
$\geqslant(3+\sqrt{5}) / 4$ by Theorem 3.6. By the concavity of $C_{\mathrm{NJ}}(\cdot, X)$, we have for all a $\in[0,1]$,

$$
C_{\mathrm{NJ}}(a, X) \geqslant(1-a) C_{\mathrm{NJ}}(0, X)+a C_{\mathrm{NJ}}(1, X) \geqslant \frac{3+\sqrt{5}+(5-\sqrt{5}) a}{4}
$$

a contradiction.

QUESTION. Is Corollary 3.13 still valid if we drop the assuption of concavity?

REMARK 3.14. In the definition of a u-space (see (2.4)), we can replace $x, y$ in $S_{X}$ by $x, y \in B_{X}$. To see this, we first observe that, $\|x\| \geqslant\|x+y\|-\|y\|$. Thus,

$$
\begin{gathered}
\text { if } x, y \in B_{X} \text { and }\left\|\frac{x+y}{2}\right\|>1-\delta \text { for some } \delta>0, \\
\text { then }\|x\| \geqslant 1-2 \delta \text { and }\|y\| \geqslant 1-2 \delta .
\end{gathered}
$$

From (3.2) if we put $x^{\prime}=x /\|x\|$ and $y^{\prime}=y /\|y\|$ we obtain

$$
\left\|\frac{x^{\prime}+y^{\prime}}{2}\right\|>1-3 \delta, \text { whenever }\left\|\frac{x+y}{2}\right\|>1-\delta .
$$

Indeed, (3.3) follows from the fact that $\left\|x^{\prime}-x\right\|<2 \delta$ and $\left\|y^{\prime}-y\right\|<2 \delta$, together with the inequality

$$
\left\|x^{\prime}+y^{\prime}\right\| \geqslant\|x+y\|-\left\|x^{\prime}-x\right\|-\left\|y^{\prime}-y\right\| .
$$

Now, given any $\varepsilon>0$, choose $\delta \in(0,(3 \varepsilon) / 4)$ so that for $x^{\prime}, y^{\prime} \in S_{X}$,

$$
\left\|\frac{x^{\prime}+y^{\prime}}{2}\right\|>1-\delta \Rightarrow f\left(y^{\prime}\right)>1-\frac{\varepsilon}{2} \text { for all } f \in \nabla_{x^{\prime}} .
$$

Then, if $x, y \in B_{X}$, and $\|(x+y) / 2\|>1-(\delta / 3),(3.3)$ implies that $\left\|\left(x^{\prime}+y^{\prime}\right) / 2\right\|>1-\delta$ where $x^{\prime}=x /\|x\|$ and $y^{\prime}=y /\|y\|$. Note, by $(3.2)$, that $\left\|y^{\prime}-y\right\|<(2 \delta) / 3$. Fix $f \in \nabla_{x}=\nabla_{x^{\prime}}$ and consider the inequalities

$$
f(y)+\frac{\varepsilon}{2}>f(y)+\frac{2 \delta}{3} \geqslant f(y)+\left\|y^{\prime}-y\right\| \geqslant f(y)+f\left(y^{\prime}-y\right)=f\left(y^{\prime}\right)>1-\frac{\varepsilon}{2} .
$$

Consequently, $f(y)>1-\varepsilon$ as required.

TheOREM 3.15. For $1<p<\infty$, all $L^{p}(\Omega)$ spaces satisfy $C_{\mathrm{N} J}\left(1, L^{p}(\Omega)\right)<2$. Indeed, all $u$-spaces $X$ have $C_{\mathrm{NJ}}(a, X)<2$ for all $0<a<2$.

Proof: Suppose $C_{\mathrm{NJ}}(2-\delta, X)=2$ for all sufficiently small $\delta>0$. For one such $\delta$ choose $x_{n}, y_{n}, z_{n} \in B_{X}$ of which at least one belongs to $S_{X}$ and such that 
$\left\|y_{n}-z_{n}\right\| \leqslant(2-\delta)\left\|x_{n}\right\|$ for each $n$ and $g\left(x_{n}, y_{n}, z_{n}\right) \nearrow 2$. Consider

$$
\begin{aligned}
g(x, y, z) & =\frac{\|x+y\|^{2}+\|x-z\|^{2}}{2\|x\|^{2}+\|y\|^{2}+\|z\|^{2}} \\
& \leqslant \frac{2\|x\|^{2}+\|y\|^{2}+\|z\|^{2}+2(\|x\|\|y\|+\|x\|\|z\|)}{2\|x\|^{2}+\|y\|^{2}+\|z\|^{2}} \\
& =1+\frac{2(\|x\|\|y\|+\|x\|\|z\|)}{2\|x\|^{2}+\|y\|^{2}+\|z\|^{2}} \leqslant 2 .
\end{aligned}
$$

This implies

$$
\frac{2\left\|x_{n}\right\|\left\|y_{n}\right\|+2\left\|x_{n}\right\|\left\|z_{n}\right\|}{2\left\|x_{n}\right\|^{2}+\left\|y_{n}\right\|^{2}+\left\|z_{n}\right\|^{2}} \rightarrow 1
$$

and then

$$
\frac{\left(\left\|x_{n}\right\|-\left\|y_{n}\right\|\right)^{2}+\left(\left\|x_{n}\right\|-\left\|z_{n}\right\|\right)^{2}}{2\left\|x_{n}\right\|^{2}+\left\|y_{n}\right\|^{2}+\left\|z_{n}\right\|^{2}} \rightarrow 0
$$

Since, for each $n$, one of $x_{n}, y_{n}, z_{n}$ belongs to $S_{X}$, we must have $\left\|x_{n^{\prime}}\right\|,\left\|y_{n^{\prime}}\right\|,\left\|z_{n^{\prime}}\right\| \rightarrow 1$ for some subsequence $\left(n^{\prime}\right)$ of $(n)$. From this, together with (3.4), one can conclude that

$$
\left\|x_{n^{\prime}}+y_{n^{\prime}}\right\|,\left\|x_{n^{\prime}}-z_{n^{\prime}}\right\| \rightarrow 2
$$

Take $f_{n^{\prime}} \in \nabla_{x_{n^{\prime}}}$ for each $n$. Since $X$ is a u-space, we have, by (3.5) and (2.4), $f_{n^{\prime}}\left(x_{n^{\prime}}-y_{n^{\prime}}\right) \rightarrow 0$ and $f_{n^{\prime}}\left(x_{n^{\prime}}+z_{n^{\prime}}\right) \rightarrow 0$. Therefore,

$$
\begin{aligned}
2\left\|x_{n^{\prime}}\right\| & =2 f_{n^{\prime}}\left(x_{n^{\prime}}\right)=f_{n^{\prime}}\left(x_{n^{\prime}}-y_{n^{\prime}}\right)+f_{n^{\prime}}\left(x_{n^{\prime}}+z_{n^{\prime}}\right)+f_{n^{\prime}}\left(y_{n^{\prime}}-z_{n^{\prime}}\right) \\
& \leqslant f_{n^{\prime}}\left(x_{n^{\prime}}-y_{n^{\prime}}\right)+f_{n^{\prime}}\left(x_{n^{\prime}}+z_{n^{\prime}}\right)+\left\|y_{n^{\prime}}-z_{n^{\prime}}\right\| \\
& \leqslant f_{n^{\prime}}\left(x_{n^{\prime}}-y_{n^{\prime}}\right)+f_{n^{\prime}}\left(x_{n^{\prime}}+z_{n^{\prime}}\right)+2-\delta .
\end{aligned}
$$

Thus, $2 \leqslant 2-\delta$ a contradiction.

REMARK 3.16.

(1) In [2], it is shown that $C_{\mathrm{NJ}}\left(L^{p}\right)=2^{(2 / t)-1}$, for $1 \leqslant p \leqslant \infty$, where $t=\min \{p, q\}$ and $(1 / p)+(1 / q)=1$. Thus, while $C_{\mathrm{NJ}}\left(L^{p}\right)$ is close to 2 for $p$ large, or near 1, Theorem 3.15 still applies and says that for $1<p<\infty$, all $L^{p}$ spaces have uniform normal structure.

(2) As a measure of uniform nonsquareness, we say $X$ is $\varepsilon$-inquadrate ( $\varepsilon$ InQ), for $0 \leqslant \varepsilon \leqslant 2$, if for any sequences $\left(x_{n}\right),\left(y_{n}\right)$ in $B_{X}$,

$$
\left\|x_{n}+y_{n}\right\| \rightarrow 2 \text { implies } \underset{n \rightarrow \infty}{\limsup }\left\|x_{n}-y_{n}\right\| \leqslant \varepsilon
$$


In [26], Tasena introduces $\varepsilon$-u-spaces and $\varepsilon$-u-smooth spaces and proves that "all $\varepsilon$-u-spaces have $C_{\mathrm{NJ}}(2-\delta, X)<2$ for all $\delta>2 \varepsilon$ ". He also observes that $\varepsilon-\operatorname{In} Q$ spaces are $\varepsilon$-u-spaces.

(3) A long standing open problem is whether $C_{\mathrm{NJ}}(0, X)<2$ implies the fixed point property. It now appears that $C_{\mathrm{NJ}}(1, X)<2$ implies uniform normal structure which in turn implies the fpp. Concerning this open problem, it is interesting to ask what is the smallest $a \in(0,1)$ for which the fpp follows whenever $C_{\mathrm{NJ}}(a, X)<2$.

\section{REFERENCES}

[1] A.G. Aksoy and M.A. Khamsi, Nonstandard methods in fixed point theory (Spinger-Verlag, Heidelberg, 1990).

[2] J.A. Clarkson, 'The von-Neumann-Jordan constant for the Lebesgue spaces', Ann. Math. 38 (1937), 114-115.

[3] M.M. Day, 'Some characterizations of inner product spaces', Trans. Amer. Math. Soc. 62 (1947), 320-337.

[4] T. Figiel, 'On the moduli of convexity and smoothness', Studia Math. 56 (1976), 121-155.

[5] J. Gao, 'Normal structure and the arc length in Banach spaces', Taiwanese J. Math. 5 (2001), 353-366.

[6] J. Gao and K.S. Lau, 'On two classes of Banach spaces with uniform normal structure', Studia Math. 99 (1991), 41-56.

[7] J. Garcia-Falset, 'The fixed point property in Banach spaces with NUS-property', J. Math. Anal. Appl. 215 (1997), 532-542.

[8] J. Garcia-Falset and B. Sims, 'Property (M) and the weak fixed point property', Proc. Amer. Math. Soc. 125 (1997), 2891-2896.

[9] K. Goebel, 'Convexivity of balls and fixed-point theorems for mappings with nonexpansive square', Compositio Math. 22 (1970), 269-274.

[10] K. Goebel and W.A. Kirk, Topics in metric fixed point theorem (Cambridge University Press, Cambridge, 1990).

[11] R.C. James, 'Uniformly non-square Banach spaces', Ann. Math. 80 (1964), 542-550.

[12] P. Jordan and J. von Neumann, 'On inner product in linear metric spaces', Ann. Math. 36 (1935), 719-723.

[13] M. Kato, L. Maligranda and Y. Takahashi, 'On James and Jordan-von Neumann constants and the normal structure coefficient of Banach spaces', Studia Math. 144 (2001), 275-295.

[14] M. Kato and Y. Takahashi, 'On the von Neumann-Jordan constant for Banach spaces', Proc. Amer. Math. Soc. 125 (1997), 1055-1062.

[15] M. Kato and Y. Takahashi, 'Von Neumann-Jordan constant for Lebesgue-Bochner spaces', J. Inequal. Appl. 2 (1998), 89-97.

[16] W.A. Kirk, 'A fixed point theorem for mappings which do not increase distances', Amer. Math. Monthly 72 (1965), 1004-1006. 
[17] W.A. Kirk and B. Sims, 'Uniform normal structure and related notions', J. Nonlinear Convex Anal. 2 (2001), 129-138.

[18] K.S. Lau, 'Best approximation by closed sets in Banach spaces', J. Approx. Theory 23 (1978), 29-36.

[19] J. Lindenstrauss and L. Tzafriri, Classical Banach spaces II. Function spaces (SpringerVerlag, Berlin, Heidelberg, New York, 1979).

[20] R.E. Megginson, An introduction to Banach space theory, Graduate Texts in Math. 183 (Springer-Verlag, New York 1998).

[21] V.D. Milman, 'Geometric theory of Banach spaces. Theory of basic and minimal systems', (Russian), Uspekhi Mat. Nauk 25 (1970), 113-174.

[22] Z. Opial, 'Weak convergence of the sequence of successive approximations for nonexpansive mappings', Bull. Amer. Math. Soc. 73 (1967), 591-597.

[23] B. Sims, "Ultra"-techniques in Banach space theory, Queen's Papers in Pure and Applied Mathematics (Queen's University, Kingston, 1982).

[24] B. Sims, 'A class of spaces with weak normal structure', Bull. Austral. Math. Soc. 49 (1994), 523-528.

[25] Y. Takahashi and M. Kato, 'Von Neumann-Jordan constant and uniformly non-square Banach spaces', Nihonkai Math. J. 9 (1998), 155-169.

[26] S. Tasena, 'On generalized u-spaces and applications to the fixed point property in Banach spaces', (preprint).

\footnotetext{
Department of Mathematics

Faculty of Science

Chiang Mai University

Chiang Mai 50200

Thailand

e-mail: sompongd@chiangmai.ac.th

g4365178@cm.edu
} 